\title{
Neonatal Sepsis: Antibiotic Sensitivity and Resistance Pattern of Most Common Isolated Pathogens in a Neonatal Intensive Care Unit of a Tertiary Care Hospital
}

\author{
Mayuri Bhise, Prakash Waghmare*, Sapana Mundhada, Anu Sharma and Kishor Ingole \\ Department of Microbiology, Dr.V.M. Government medical college Solapur, Maharashtra, India \\ *Corresponding author
}

\section{A B S T R A C T}

\begin{tabular}{|l|}
\hline K e y w o r d s \\
Neonatal septicemia, \\
Blood culture, Antibiotic \\
$\begin{array}{l}\text { Susceptibility Pattern, } \\
\text { Extended Spectrum } \beta- \\
\text { Lactamases (ESBL), } \\
\text { Methicillin Resistant } \\
\text { Staphylococcus aureus } \\
\text { (MRSA). }\end{array}$ \\
\hline Article Info \\
\hline Accepted: \\
23 October 2017 \\
Available Online: \\
10 December 2017 \\
\hline
\end{tabular}

Septicemia in neonates refers to generalized bacterial infection documented by positive blood culture in the first four weeks of life. Neonatal sepsis is one of the commonest causes of neonatal mortality in the developing world. To isolate and identify the bacterial isolates responsible for neonatal sepsis. Objective of the study is to determine the susceptibility pattern of isolates. Isolates wereidentified.Antibiotic susceptibility of the isolates was done by Kirby Bauer disc diffusion method according to CLSI guidelines 2014. Methicillin resistant Staphylococcus aureus (MRSA) and ESBL detection were also done. Among the culture positive specimens, Klebsiella pneumoniae was the most commonly isolated pathogen followed by Escherichia coli and overall Gram negative organisms were isolated predominantly than gram positive organisms Gram negative bacteria also exhibited high resistance to the commonly prescribed group of drugs such as penicillins and cephalosporins, Klebsiella pneumoniae and MRSA were the commonly isolated gram negative and gram positive organism respectively.

\section{Introduction}

Septicaemia in neonates refers to generalized bacterial infection documented by positive blood culture in the first four weeks of life(1) and is one of the four leading causes of neonatal mortality and morbidity in India. $(2,3,4)$ Neonatal septicaemia continues to be a major problem for neonates in neonatal intensive care units around the world.(5)

Neonatal mortality rate is one of the indicators for measuring the health status of a nation.(6) There could be various reasons for neonatal mortality but septicaemia continues to be a major cause of neonatal mortality and morbidity worldwide. Incidence varies from country to country, but it is much higher in developing countries than in developed nations.(6) According to World Health Organization (WHO) estimates, there are about 5 million 'neonatal deaths a year, with $98 \%$ occurring in developing countries.(7)

Neonatal sepsis is broadly divided into two types according to age of onset: Early-onset sepsis ( $<72 \mathrm{hrs}$ ) and late-onset sepsis $(\geq 72$ hrs-28 days). Early-onset sepsis is acquired during fetal life, delivery, or at the nursery.(8) Neonatal sepsis is caused by a variety of Gram-positive as well as Gram-negative bacteria, and sometimes yeasts.(5) The spectrum of organisms that causes neonatal sepsis changes over times and varies from region to region. This is due to the changing 
pattern of antibiotic use and changes in lifestyle.(9)

Periodic evaluation of organisms responsible for neonatal sepsis is essential for the appropriate management of neonates. Therefore, this study was undertaken to determine the profile and antibiotic sensitivity patterns of aerobic isolates from blood cultures of neonates in a tertiary care hospital

The main aim of this study to isolate and identify the bacterial isolates responsible for neonatal sepsis and to determine the susceptibility pattern of isolates.

\section{Materials and Methods}

A descriptive study was conducted in the department of Microbiology in a tertiary care hospital.

All neonates of either sex admitted in NICU during the period of July 2015 and July 2016 with altered body temperature, tachypnoea/apnoea, lack of activity, poor feeding, abdominal distension, jaundice, irritability and convulsion etc were clinically diagnosed to have septicaemia were included in the study. Fully informed and voluntary consents were obtained from the parents or attendants. Detailed history and complete physical examinations of each neonate was carried out and recorded Blood sample was collected for culture with proper aseptic precautions. The local site was cleansed with $70 \%$ alcohol and povidone iodine (1\%), followed by $70 \%$ alcohol again before initiating antibiotic therapy, whenever possible. Approximately 1-3 ml of blood was collected in sterile bottle containing $1 \%$ glucose broth and incubated at $37^{\circ} \mathrm{C}$. Blind subculture were made on Blood agar, Chocolate agar and MacConkey agar after 24 hours, 48 hours, 72 hours and 7 days, If no growth was observed on plates after 7 th day, the sample was reported as negative. Isolate was identified by their characteristic appearance on their respective media, Gram staining and confirmed by the pattern of biochemical reactions.(10) Members of the family Enterobacteriaceae were identified by indole production, $\mathrm{H}_{2} \mathrm{~S}$ production, citrate utilization, motility test, urease test, oxidase, carbohydrate utilization tests, and other tests. For Gram-positive bacteria, coagulase, catalase, bacitracin and optochin susceptibility tests and other tests were used. Blood culture broth that showed no microbial growth within seven days was reported as culture negative, only after result of routine subculture on Blood, MacConkey, and chocolate agar (10). Antimicrobial susceptibility testing was performed for all blood culture isolates by Kirby-Bauer disc diffusion method according to CLSI guidelines 2014 (11).

Methicillin resistant Staphylococcus aureus (MRSA) detection was done according to CLSI guidelines 2014 using cefoxitin disc $(30 \mathrm{~g})$. The plates were incubated for 24 hours at $35 \mathrm{C}$ and zone diameter was measured. Ifzone diameter was $22 \mathrm{~mm}$, it was considered as Methicillin sensitive Staphylococcus aureus (MSSA) and $21 \mathrm{~mm}$ then it was considered as MRSA. The ESBL detection in case of E. coli and $K$. pneumoniae isolates was done using phenotypic confirmatory method as per CLSI guidelines.(11) An isolate was considered as ESBL producer when zone diameter of ceftazidime/clavulanic acid disc $(30 / 10 \mu \mathrm{g})$ was $5 \mathrm{~mm}$ than the diameter of ceftazidime $(30 \mu \mathrm{g})$.

The drugs for disc diffusion testing were in the following concentrations: Ampicillin (10 $\mu \mathrm{g})$, amoxiclav $(20 / 10 \mu \mathrm{g})$, ciprofloxacin (5 $\mu \mathrm{g})$, erythromycin $(15 \mu \mathrm{g})$, gentamicin (10 $\mu \mathrm{g})$, penicillin (10 units), co-trimoxazole (1.25 $\mu \mathrm{g}$ trimethoprim/23.75 $\mu$ gsulfamethoxazole), amikacin (30 $\mu \mathrm{g})$, ceftriaxone, ceftazidime + clavulanic acid, imepenum 
(10ug). The discs were obtained from Himedia (India) Laboratories.

\section{Results and Discussion}

Out of 80 patients admitted, 60 patients were male and 20 patients were female. Empirical antibiotic therapy was started in all neonates with risk factors suggestive of sepsis before the results of blood culture susceptibility. 40 (50\%) out of 80 patients admitted during the study period had proven sepsis confirmed by positive blood culture. Among the culture positive specimens, Klebsiella pneumoniae $(52.6 \%)$ was the most commonly isolated pathogen followed by Escherichia coli (12.5\%), Acinetobacter baumannii (5\%) and Gram negative organisms accounted for 72.5 $\%$ and Gram positive accounted for $27.5 \%$ of all positive cultures. Klebsiella pneumoniae and MRSA were the commonly isolated gram negative and gram positive organism respectively.

It was seen in table 1 and chart 1 that out of 40 culture positive neonatal patients, 26 $(65 \%)$ were male and $14(35 \%)$ were female.

Gram negative bacilli (isolates) were predominantly isolated from suspected septicemia patients as compared to gram positive cocci (isolates) (Table 2).

The uncertainty surrounding the clinical approach to treatment of neonatal septicemia can be minimized by periodic epidemiological surveys of aetiological agents and their antibiotic sensitivity patterns leading to recognition of the most frequently encountered pathogens in a particular geographical area. For effectual management of septicemia cases, study of bacteriological profile along with the antimicrobial sensitivity pattern plays a noteworthy role. $(12,13,14)$ Out of the 80 clinically suspected cases of sepsis in our study, 40 were culture positive with a blood culture positivity rate of $50 \%$. The incidence of Gram-negative and Grampositive organisms was $65 \%$ and $35 \%$, respectively. There were $24(60 \%)$ isolates from early onset septicemia cases, while 16 $(40 \%)$ were from late-onset illness (Table 3 and 4).

Male preponderance was observed in the present study which is in concordance with the above referred studies. The possible reason for the male preponderance is that the factors regulating synthesis of gamma globulins are probably situated on $\mathrm{X}$ chromosome and presence of one $\mathrm{X}$ chromosomes in male thus confers less immunological protection compared to female counter part.(15) Culture-positivity for aerobic organisms in neonates vary from $25 \%$ to $60 \%(15,16,17)$. In this study, blood culture-positivity rate is $50 \%$. This finding is comparable with other reports.(9) However, a high blood culture-positivity rate in septicemic children (56\%) had been reported by Sharma et al.,(18) and Jain et al.,(19)

A low blood culture isolation rate could be due to administration of antibiotic before blood collection from the primary centers or the possibility of infection with anaerobes.

A negative blood culture does not exclude sepsis and about $26 \%$ of all neonatal sepsis could be due to anaerobes.(9)

The pathogens most often implicated in neonatal sepsis in developing countries differ from those seen in developed countries. 
Table.1 Distribution of cases according to sex

\begin{tabular}{|l|l|l|}
\hline Sex & Total number & Percentage \\
\hline Male & 26 & 65 \\
\hline Female & 14 & 35 \\
\hline Total & 40 & 100 \\
\hline
\end{tabular}

Table.2 Showing distribution of blood culture positivity

\begin{tabular}{|l|l|l|l|}
\hline Number of patients & $\begin{array}{l}\text { Blood Culture } \\
\text { Positive }\end{array}$ & $\begin{array}{l}\text { Blood Culture } \\
\text { Negative }\end{array}$ & Total \\
\hline$<28$ days (neonates) & $\mathbf{4 0}(\mathbf{5 0 \%})$ & $\mathbf{4 0}(\mathbf{5 0 \%})$ & $\mathbf{8 0}(\mathbf{1 0 0 \%})$ \\
\hline
\end{tabular}

Table.3 Bacterial isolates (gram positive and gram negative isolates) in suspected septicemia in neonatal patients $(n=40)$

\begin{tabular}{|l|c|c|}
\hline Isolates & Total isolates $(\mathbf{n}=\mathbf{4 0})$ & Percentage \\
\hline Gram negative bacilli & 26 & 65 \\
\hline Gram positive cocci & 14 & 35 \\
\hline
\end{tabular}

Table.4 Frequency of bacterial isolates causing septicaemia

\begin{tabular}{|l|l|c|c|}
\hline Sr. No. & Organism & Number & Percentage \\
\hline A & Gram Positive Isolates & $\mathbf{1 4}$ & $\mathbf{3 5}$ \\
\hline 1 & Staphylococcus. aureus & 8 & 20 \\
\hline 2 & $\begin{array}{l}\text { Coagulase Negative Staphylococcus } \\
\text { aureus }\end{array}$ & 3 & 7.5 \\
\hline B & Gram Negative Isolates & $\mathbf{2 6}$ & $\mathbf{6 5}$ \\
\hline 1 & Klebsiella pneumoniae & 21 & 52.6 \\
\hline 2 & Escherichia coli & 5 & 12.5 \\
\hline 3 & Acinetobacter baumannii & 2 & 5 \\
\hline 4 & Pseudomonas aeruginosa & 1 & 2.5 \\
\hline
\end{tabular}

Table.5 Blood culture positive result showing EOS and LOS among neonates

\begin{tabular}{|l|l|}
\hline $\begin{array}{l}\text { Septicemia among neonates }(<\mathbf{2 8} \text { days }) \\
(\mathbf{n}=\mathbf{4 0})\end{array}$ & Blood Culture Positive \\
\hline Early onset Septicemia & $24(60 \%)$ \\
\hline Late onset septicemia & $16(40 \%)$ \\
\hline Total & $40(100 \%)$ \\
\hline
\end{tabular}



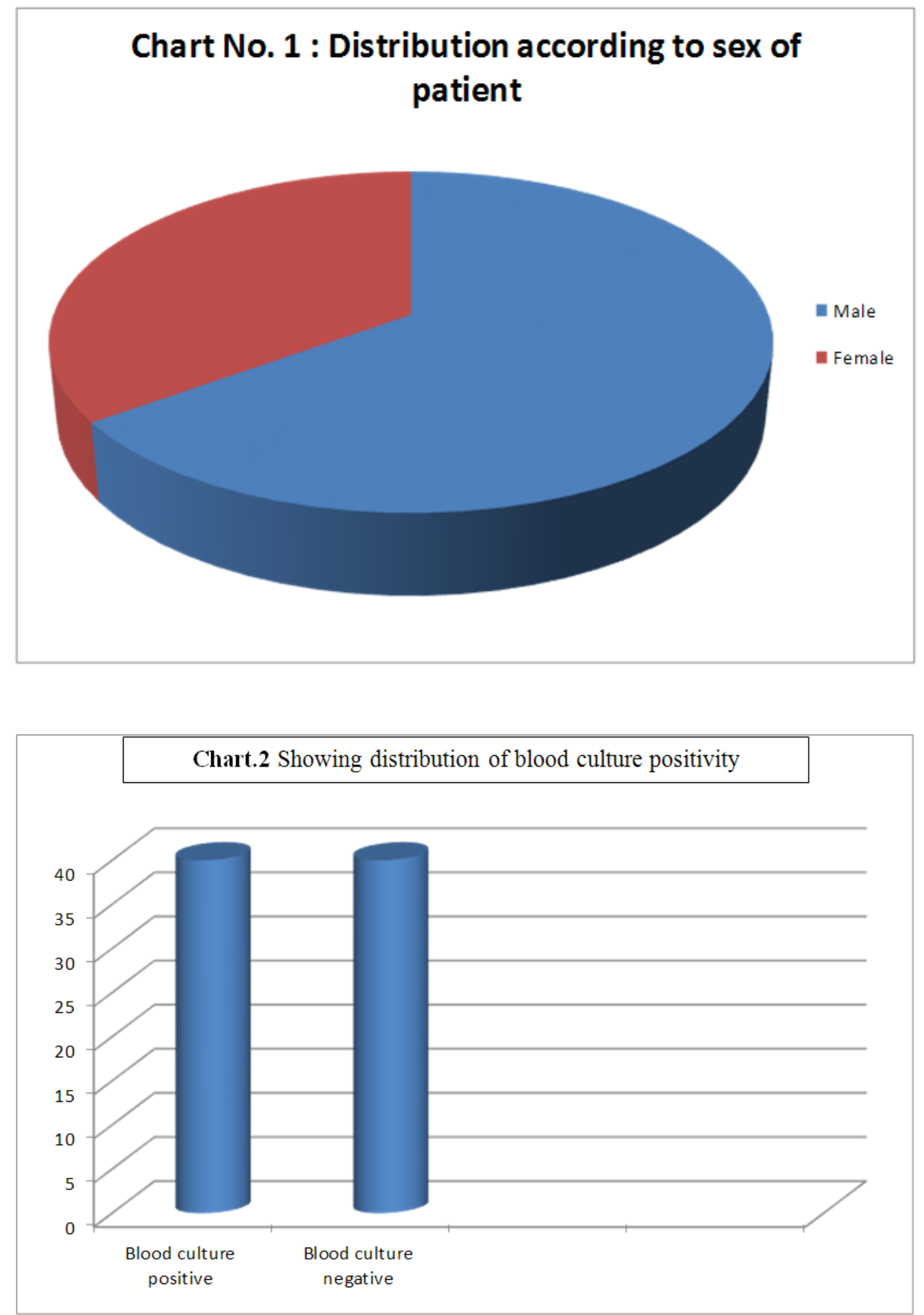


\section{Chart No. 3: Distribution among total Blood culture positive neonatal isolates $(n=40)$}

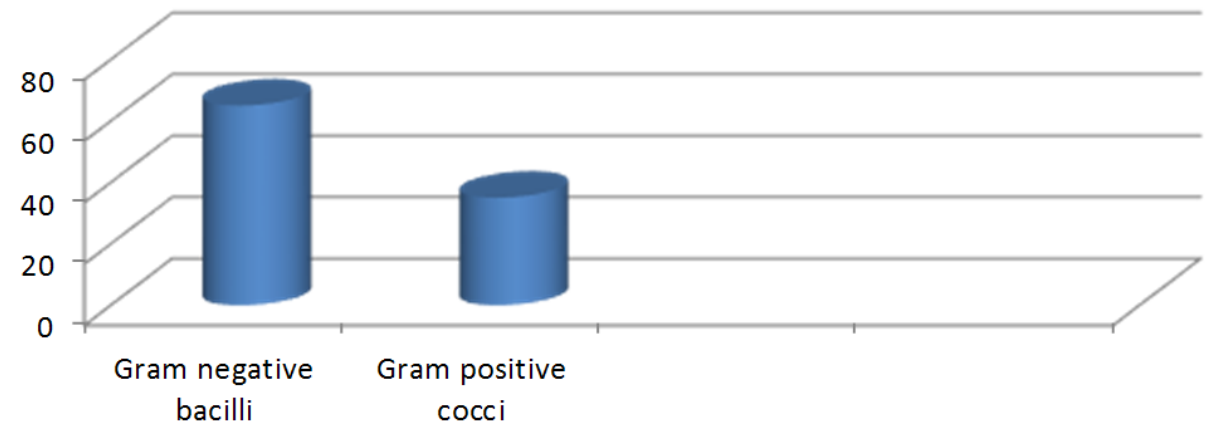

\section{Chart No. 4 : Distribution of Isolates}

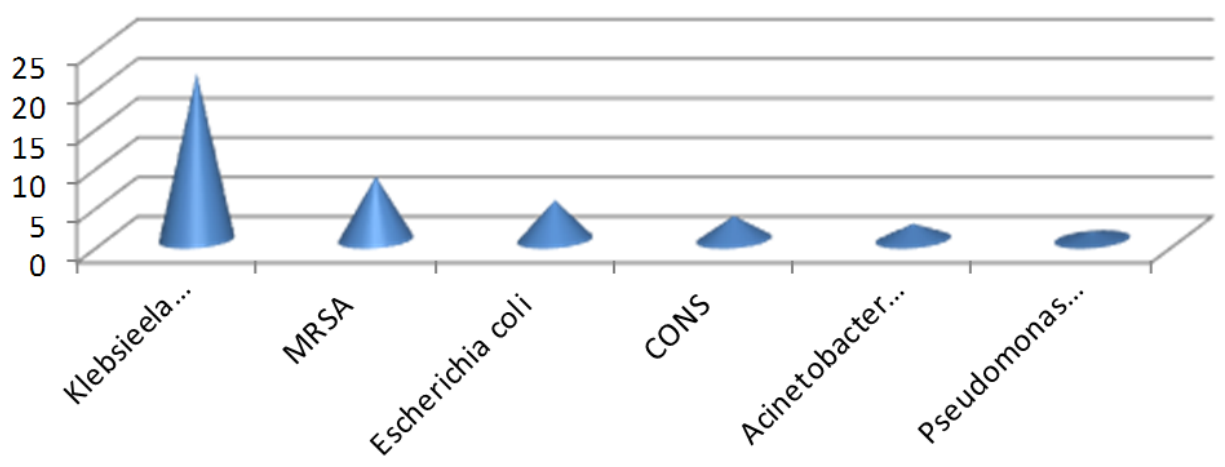

Chart no 5: Distribution of EOS and LOS among neonates who are blood culture positive $(n=40)$

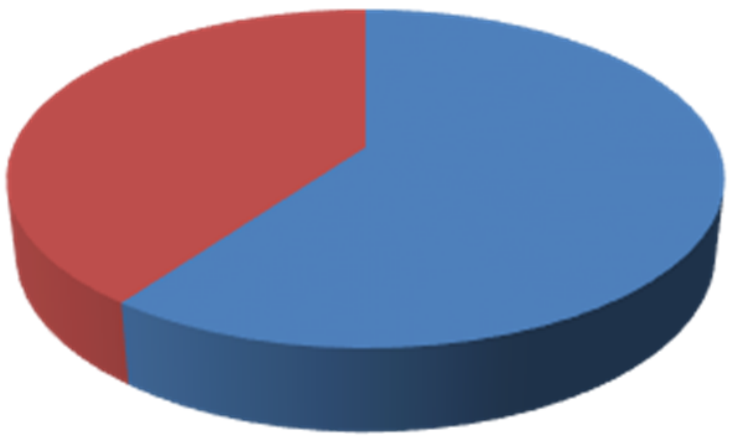

Early onset Septicemia

- Late onset septicemia 
Overall, Gram-negative organisms are more common and are mainly represented by Klebsiella pneumoniae, Escherichia coli, Acinetobacter baumanii, Pseudomonas, and Salmonella. In Gram-positive organisms, Staphylococcus aureus, CONS are most commonly isolated (7).

Gram-negative and Gram-positive septicemia was encountered in $72.5 \%$ and $27.5 \%$ of the culture-positive cases in this study, which is comparable to a study conducted by Agnihotri et al., (1) which reported that Gram-negative and Gram-positive organisms were responsible for $72 \%$ and $27 \%$ of the septicemia cases, respectively. Similar observations were made by other workers (4, 6).

The report of the National Neonatal-Perinatal database showed Klebsiella pneumoniaeas the predominant $(29 \%)$ pathogen (15). Klebsiella pneumoniae. (52.6\%) was the predominant Gram-negative species isolated in this study, which agrees with previous reports $(2,9)$. Of the total 80 cases of neonatal sepsis, 24 (60\%) were early-onset sepsis in this study, which is comparable to previous studies (Table.5) $(1,6)$.

Antibiotic resistance is today a global problem. Reports of multi-resistant bacteria causing neonatal sepsis in developing countries are increasing. The wide availability of over-the-counter antibiotics and the inappropriate use of broad-spectrum antibiotics in the community may explain this situation. It is difficult to compare antibiotic resistance between countries because the epidemiology of neonatal sepsis is extremely variable.(7)

Antibiotic susceptibility pattern was studied for all isolates causing neonatal sepsis. The analysis of drug resistance pattern showed that, among Gram-negative isolates, maximum numbers $(97 \%)$ were resistant to ampicillin and lowest to imipenem (7\%) Resistance was observed to be against commonly used antibiotics such as penicillin and third generation cephalosporins. Among Gram-positive isolates, high resistance was seen to penicillin and ampicillin (90\%). Moderate level of resistance was seen with ciprofloxacin and erythromycin.

The greater prevalence of resistance to commonly used antibiotics has also been reported by other studies. $(2,4)$ Among aminoglycosides, amikacin was found to have an edge over gentamicin in Gram-negative septicemia, with sensitivity of $52 \%$, and $33 \%$, respectively. Similar observations have been made by previous group of workers.(20)

In this study, maximum sensitivity (93\%) was observed in imipenem and vancomycin $(91 \%)$. Sensitivity to imipenem and vancomycin was much higher than that to other antibiotics and the difference was statistically significant, but these two drugs should not be used indiscriminately and be kept as a reserve drugs, otherwise resistance to these drugs may develop, thereby threatening the treatment.

Neonatal septicemia is associated with high morbidity and mortality. In the present study mortality rate was $15 \%$ in blood culture positive cases. There was significant statistical association between mortality and blood culture positivity.

Klebsiella pneumoniae was the predominant organism isolated from $3(7.5 \%)$ cases followed by Staphylococcus aureus 2 (5\%), Pseudomonas aeruginosa 1 (2.5\%) cases of mortality. The findings were consistent with Reddy et al., (21) which also showed Klebsiella species as a major cause of deaths amongst neonates. It is evident from this study that Gram-negative organisms (Klebsiella pneumoniae), and Gram positive organisms (MRSA) are the leading cause of 
neonatal sepsis in this study, and most of them are resistant to multiple antibiotics. Therefore, surveillance of antimicrobial resistance is necessary. Also, an antibiotic policy should be formulated in the hospital. Depending on the antibiotic sensitivity pattern of the isolates, antibiotics should be used. Furthermore, health education should be provided to the public on the dangers of indiscriminate use of antibiotics, which is currently considered to be a menace in our society and which has been responsible for the ineffectiveness of most commonly used antibiotics, as observed in our study.

\section{References}

1. Agnihotri N, Kaistha N, Gupta V. Antimicrobial susceptibility of isolates from neonatal septicemia. Jpn J Infect Dis. 2004; 57: 273-5.

2. Tsering DC, Chanchal L, Pal R, Kar S. Bacteriological profile of septicemia and the risk factors in neonates and infants in Sikkim. J Global Infect Dis. 2011; 3: 425.

3. Jain A, Awasthi AK, Kumar M. Etiological and antimicrobial susceptibility profile of nosocomial blood stream infections in a neonatal intensive care unit. Indian $\mathbf{J}$ Med Microbiol. 2007; 25: 299-300.

4. Kumhar GD, Ramachandran VG, Gupta P. Bacteriological analysis of blood culture isolates from neonates in a tertiary care hospital in India. J Health PopulNutr. 2002; 20: 343-7.

5. Gomaa HHA, Udo EE, Rajaram U. Neonatal septicemia in Al-Jahra hospital, Kuwait: Etiologic agents and antibiotic sensitivity patterns. Med PrincPract. 2001; 10: 145-50.

6. Kaistha N, Mehta M, Singla N, Garg R, Chander J. Neonatal septicemia isolates and resistance patterns in a tertiary care hospital of North India. J Infect DevCtries. 2009; 4: 55-7.

7. Vergnano S, Sharland M, Kazembe P, Mwansambo C, Heath PT. Neonatal sepsis: An international perspective. Arch Dis Child Fetal Neonatal Ed. 2005; 90: F220-4.

8. Puopolo KM. Bacterial and fungal infection. In: Cloherty JP, Eichenwald EC, Stark AR, editors. Manual of neonatal care. 6th ed. Philadelphia: Lippincott William and Wilkins; 2008. pp. 274-300.

9. Shrestha P, Das BK, Bhatta NK, Jha DK, Das B, Setia A, et al., Clinical and bacteriological profiles of blood culture positive sepsis in newborns. J Nepal Paediatr Soc. 2008; 27: 64-7.

10. Collee JG, Marr W. Culture of Bacteria. In: Collee JG, Fraser AG, Marmion BP, Simmons A, editors. Mackie and McCartney Practical Medical Microbiology. 14th edn. New York: Churchill Livingstone; 1996. pp. 11329.

11. Wayne, PA: NCCLS; 2014. National Committee for Clinical Laboratory Standards. Performance standards for antimicrobial disc susceptibility testing. Fourteenth informational supplement (M100-S14).

12. Zakariya BP, Bhat V, Harish BN, ArunBabu T, Joseph NM. Neonatal sepsis in a tertiary care hospital in South India: Bacteriological profile and antibiotic sensitivity pattern. Indian $\mathrm{J}$ Pediatr. 2011; 78: 413-7

13. Dutta S, Reddy R, Sheikh S, Kalra J, Ray P, Narang A. Intrapartum antibiotics and risk factors for early onset sepsis. Arch Dis Child Fetal Neonatal Ed. 2010; 95: F99-103.

14. Jiang JH, Chui NC, Huang FY, Kao HA, Hsu CH, Hung HY, et al., Neonatal sepsis in the neonatal intensive care unit: Characteristics of early versus late 
onset. J Microbial Immunol Infect. 2004; 37: 301-6.

15. Neonatal morbidity and mortality; report of the National NeonatalPerinatal Database. Indian Pediatr.1997; 34: 1039-42.

16. Mathur NB. Neonatal sepsis. Indian Pediatr. 1996; 33: 663-74.

17. Mathur M, Shah H, Dixit K, Khambadkone S, Chakrapani A, Irani S. Bacteriological profile of neonatal septicemia cases (for the year 1990-91) J Postgrad Med. 1994; 40: 18-20.

18. Sharma PP, Halder D, Dutta AK, Dutta $\mathrm{R}$, Bhatnagar S, Bali A, et al., Bacteriological profile of neonatal septicemia. Indian Pediatr. 1987; 24: 1011-7
19. Jain NK, Jain VM, Maheshwari S. Clinical profile of neonatal sepsis. Kathmandu Univ Med J. 2003; 1: 11720.

20. Bhat YR, Lewis LE, Vandana KE. Bacterial isolates of early-onset neonatal sepsis and their antibiotic susceptibility pattern between 1998 and 2004: An audit from a center in India. Ital J Pediatr. 2011; 37: 32.

21. Reddy R, Pathania S, Kapil A, Bakhshi S. Review of spectrum and sensitivity of bacterial bloodstream isolates in children with malignancy: A retrospective analysis from a single center. Indian J Cancer 2014; 51: 425-7.

\section{How to cite this article:}

Mayuri Bhise, Prakash Waghmare, Sapana Mundhada, Anu Sharma and Kishor Ingole. 2017. Neonatal Sepsis: Antibiotic Sensitivity and Resistance Pattern of Most Common Isolated Pathogens in a Neonatal Intensive Care Unit of a Tertiary Care Hospital. Int.J.Curr.Microbiol.App.Sci. 6(12): 3040-3048. doi: https://doi.org/10.20546/ijcmas.2017.612.355 